\title{
INTRODUCING AN EFFECTIVE NANOCATALYTIC FOR THE ONE-POT SYNTHESIS AND INVESTIGATION OF BIOLOGICAL PROPERTIES OF PYRANOPYRIMIDINONE AND XANTHENE DERIVATIVES
}

\author{
A. AMININIA ${ }^{a}$, K. POURSHAMSIAN ${ }^{a^{*}}$ AND B. SADEGHI ${ }^{a}$ \\ ${ }^{a}$ Department of Chemistry, Tonekabon branch, Islamic azad university, Tonekabon, Iran.
}

\begin{abstract}
In this study, an effective method for the synthesis of pyranopyrimidinone and xanthene derivatives is introduced. In this method, zinc oxide-chitosan nanocomposite has been used as a catalyst and all reactions were performed under irradiated in a microwave oven at a power of $230 \mathrm{~W}$. The properties of zinc oxide-chitosan nanocomposite were characterized by X-ray diffraction (XRD), energy dispersive X-ray (EDAX) analysis and scanning electron microscopy (SEM). The results indicated that the size of zinc oxide-chitosan nanocomposite varies between $76-95 \mathrm{~nm}$. The catalyst activity has maintained its activity after four runs. The main advantages of this method are; Eco-friendly procedure, catalytic synthesis of affordable and inexpensive materials, short reaction time, and excellent yields which makes it more economic than the other conventional methods. The antibacterial activity of the products was investigated using Minimum Inhibitory Concentration (MIC) and the Minimum Bactericidal Concentration (MBC) methods. Pyranopyrimidinone derivatives exhibit more antibacterial activity.
\end{abstract}

Keywords: Zinc oxide-chitosan nanocomposite, microwave, pyranopyrimidinone, xanthene, Biological activity, MIC, MBC.

\section{INTRODUCTION}

Pyranopyrimidinone and xanthene derivatives are widely used in pharmaceutical chemistry [1]. This group of organic compounds have various pharmaceutical activities such as antiviral2, antibacterial3, antitumor [4], hepatoprotective [5], antifungal activities [6], in the food industry as additives [7] and anti-inflammatory properties [8]. In order to synthesize this class of compounds, several methods have been reported, including the reaction of the palladium-catalysed cyclisation [9-12], different Lewis acids such as $\mathrm{NbCl} 2$ [13$16]$ and Brønsted acids [17-20].

Many of the reported methods for synthesizing these compounds have limitations, including strong acidic conditions, long reaction times, low yields, N2 atmosphere, expensive reagents and toxic organic solvents [21-25]. Given the limitations, it seems that the introduction of a more appropriate, new and effective high-activity catalyst for the synthesis of these important drug compounds is necessary.

In almost all organic reactions that require heat to produce the product, traditional heating methods such as an oil bath or a sand bath are used. These methods cause certain problems, such as sudden rise in temperature or temperature constraints, which can lead to product decomposition or the production of side products. While heating up the reaction by microwave, in addition to solving the problems created by heating in the traditional ways can be very rapid and generate a heat system that cannot be easily accessed by other methods [26-30]. The main advantage of using microwave in synthesis of organic compounds is the shorter reaction times.

The goal of this study is introduction of efficient method using a new catalyst with high activity, shorten the reaction time, recyclability, and simple reaction working-up for the preparation of pyranopyrimidinone and xanthene derivatives under neutral, mild and practical conditions.

\section{RESULTS AND DISCUSSION}

In continuation of our previous studies [31-37], our research team has tried to introduce a simple and effective method for the synthesis of pyrano[2,3d]pyrimidine and xanthen derivatives.

The results show that the use of the $\mathrm{ZnO}-\mathrm{Ch} \mathrm{NCs}$ as a catalyst with microwave radiation significantly increases the reaction speed. Also, the catalyst produced a good yield on products in neutral conditions and in ecofriendly solvents. In the conditions, the working-up of the reaction is also very easy. (Schemes 1 and 2).

The morphology of the ZnO-Ch NCs was investigated with a scanning electron microscopy (SEM). Figure 1 (left) illustrated that the $\mathrm{ZnO}-\mathrm{Ch}$ NCs was with an average size of $85 \mathrm{~nm}$. SEM image at higher magnification is also shown in Figure 1 (right).

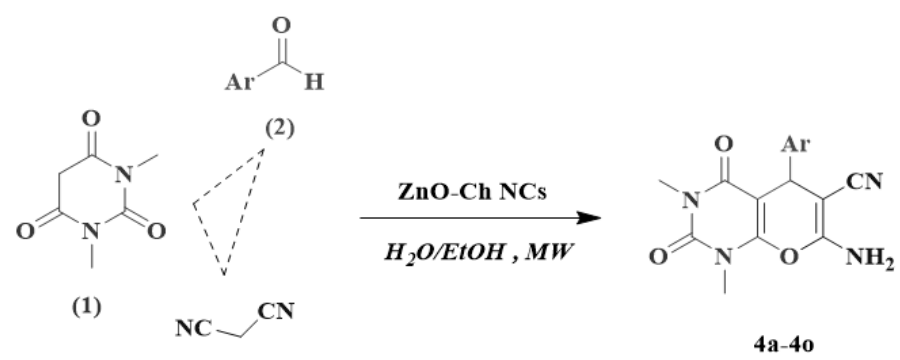

(3)

Scheme 1. Synthesis of pyrano[2,3-d]pyrimidine derivatives.<smiles>Oc1ccc2ccccc2c1</smiles>

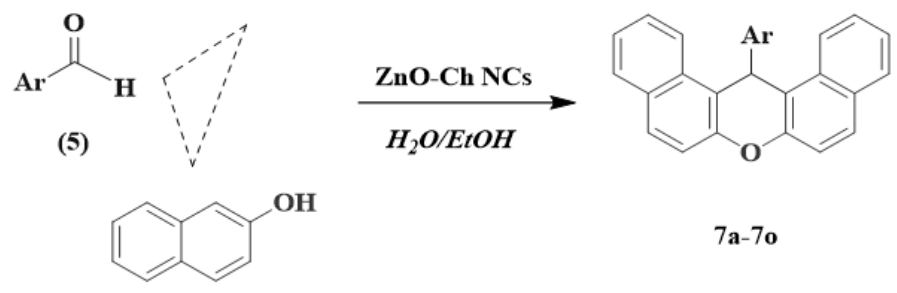

(6)

Scheme 2. Synthesis of xanthene derivatives.

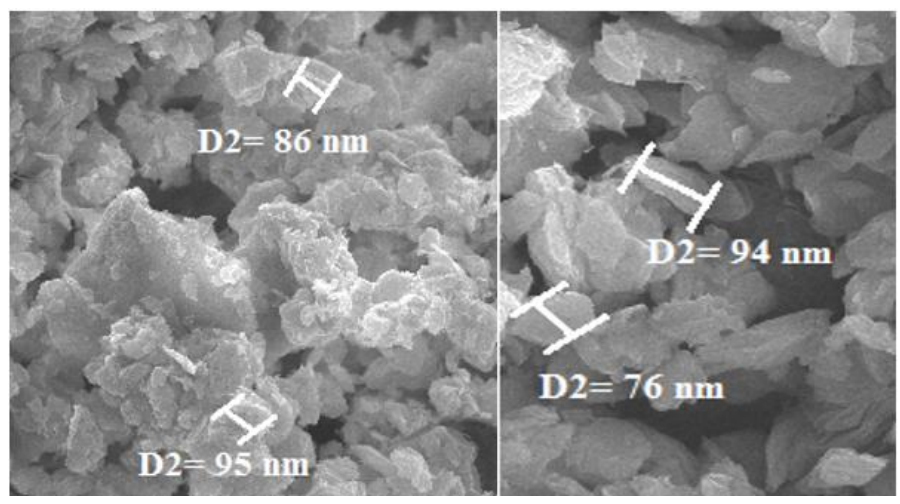

Figure 1. SEM images of a $\mathrm{ZnO}-\mathrm{Ch}$ NCs. higher magnification; View field: $3.5 \mu \mathrm{m}$ (right), lower magnification; View field: $8.5 \mu \mathrm{m}$ (left). 
The chemical purity of the samples was tested by EDAX spectra. The EDAX spectra given in Figure 2 shows the presence of zinc, oxygen, carbon and nitrogen as the only elementary components.

The X-ray diffraction patterns (XRD) of the ZnO-Ch NCs is shown in Figure 2. XRD study of $\mathrm{ZnO}-\mathrm{Ch}$ NCs confirmed presence of $\mathrm{ZnO}$ in the $\mathrm{ZnO}-\mathrm{Ch} \mathrm{NCs}$ (Figure 2)
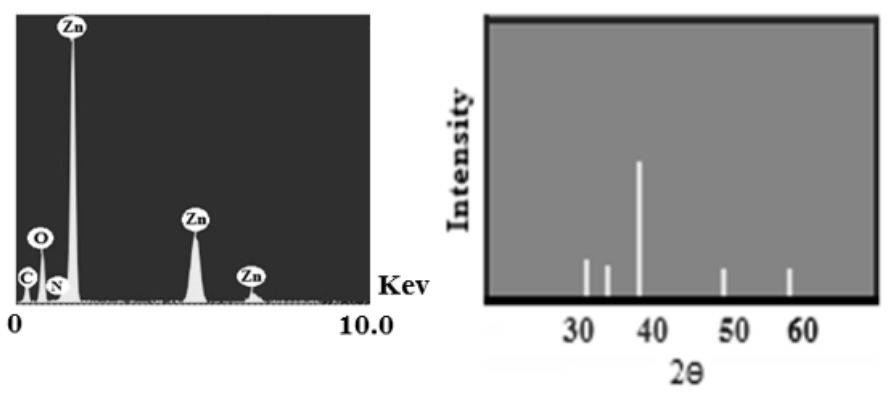

Figure 2. EDAX spectra of $\mathrm{ZnO}-\mathrm{Ch} \mathrm{NCs}$ (left) and XRD patterns of $\mathrm{ZnO}$ starch nanocomposite (right).

Initially, in order to find optimal conditions for the synthesis of pyranopyrimidinone and xanthene derivatives, reactions were designed in different conditions. The results are tabulated in Tables 1 and 2. The optimized reaction conditions are shown with red pen (Table 1 entry 13 and Table 2, entry $11)$.

In scheme 3, a proposed mechanism for the synthesis of pyranopyrimidinone and xanthene derivatives in the presence of a catalyst was introduced. In synthesis process of pyranopyrimidinone derivatives, initially via a Knoevenagel reaction of activated malononitrile and activated aromatic aldehyde was formed the yellowish arylidenemalononitrile in a quantitative yield. The formation of the pyranopyrimidinone derivatives is shown in scheme 3 .

Table 1. Effect of temperature, amount of the catalyst and solvent on the synthesis of $4 \mathrm{a}$ in the presence of $\mathrm{ZnO}$-Ch NCs.

\begin{tabular}{|c|c|c|c|c|c|}
\hline Entry & $\begin{array}{c}\text { Catalyst } \\
\text { amount } \\
(\mathrm{mg})\end{array}$ & Solvent & Temperature $\left({ }^{\circ} \mathrm{C}\right)$ & $\begin{array}{l}\text { Time } \\
\text { (min) }\end{array}$ & $\begin{array}{c}\text { Yield } \\
(\%)\end{array}$ \\
\hline 1 & 5 & $\mathrm{H}_{2} \mathrm{O}$ & MW & 10 & - \\
\hline 2 & 10 & $\mathrm{H}_{2} \mathrm{O}$ & MW & 10 & 33 \\
\hline 3 & 15 & $\mathrm{H}_{2} \mathrm{O}$ & MW & 10 & 54 \\
\hline 4 & 20 & $\mathrm{H}_{2} \mathrm{O}$ & MW & 10 & 72 \\
\hline 5 & 25 & $\mathrm{H}_{2} \mathrm{O}$ & MW & 10 & 75 \\
\hline 6 & 27 & $\mathrm{H}_{2} \mathrm{O}$ & MW & 10 & 80 \\
\hline 7 & 28 & $\mathrm{H}_{2} \mathrm{O}$ & MW & 10 & 84 \\
\hline 8 & 30 & $\mathrm{H}_{2} \mathrm{O}$ & MW & 10 & 85 \\
\hline 9 & 35 & $\mathrm{H}_{2} \mathrm{O}$ & MW & 10 & 86 \\
\hline 10 & 28 & $\mathrm{EtOH}$ & MW & 10 & 80 \\
\hline 11 & 28 & DMF & MW & 10 & 61 \\
\hline 12 & 28 & $\mathrm{CH}_{2} \mathrm{Cl}_{2}$ & MW & 10 & 70 \\
\hline 13 & 28 & $\begin{array}{c}\mathrm{EtOH} / \mathrm{H}_{2} \mathrm{O} \\
(1 / 1)\end{array}$ & MW & 10 & 93 \\
\hline 14 & 28 & $\mathrm{H}_{2} \mathrm{O}$ & Reflux & 45 & 79 \\
\hline 15 & 28 & $\mathrm{EtOH} / \mathrm{H}_{2} \mathrm{O}(1 / 1)$ & Reflux & 25 & 77 \\
\hline 16 & 28 & $\mathrm{EtOH} / \mathrm{H}_{2} \mathrm{O}(1 / 1)$ & Reflux & 35 & 81 \\
\hline 17 & 28 & $\mathrm{EtOH} / \mathrm{H}_{2} \mathrm{O}(1 / 1)$ & Reflux & 45 & 87 \\
\hline 18 & 28 & $\mathrm{EtOH} / \mathrm{H}_{2} \mathrm{O}(1 / 1)$ & Reflux & 60 & 92 \\
\hline 19 & 28 & Solvent free & MW & 60 & 38 \\
\hline
\end{tabular}

Table 2. Effect of temperature, amount of the catalyst and solvent on the synthesis of $7 \mathrm{c}$ in the presence of $\mathrm{ZnO}$-Ch NCs.

\begin{tabular}{|c|c|c|c|c|c|}
\hline Entry & $\begin{array}{c}\text { Catalyst amount } \\
(\mathrm{mol} \%)\end{array}$ & Solvent & $\begin{array}{c}\text { Temperature } \\
\left({ }^{\circ} \mathrm{C}\right)\end{array}$ & $\begin{array}{l}\text { Time } \\
(\text { min) }\end{array}$ & $\begin{array}{c}\text { Yield } \\
(\%)\end{array}$ \\
\hline 1 & 5 & $\mathrm{H}_{2} \mathrm{O}$ & MW & 15 & 38 \\
\hline 2 & 10 & $\mathrm{H}_{2} \mathrm{O}$ & MW & 15 & 57 \\
\hline 3 & 15 & $\mathrm{H}_{2} \mathrm{O}$ & MW & 15 & 60 \\
\hline 4 & 20 & $\mathrm{H}_{2} \mathrm{O}$ & MW & 15 & 69 \\
\hline 5 & 25 & $\mathrm{H}_{2} \mathrm{O}$ & MW & 15 & 72 \\
\hline 6 & 30 & $\mathrm{H}_{2} \mathrm{O}$ & MW & 15 & 81 \\
\hline 7 & 35 & $\mathrm{H}_{2} \mathrm{O}$ & MW & 15 & 83 \\
\hline 8 & 30 & $\mathrm{EtOH}$ & MW & 15 & 79 \\
\hline 9 & 30 & DMF & MW & 15 & 65 \\
\hline 10 & 30 & $\mathrm{CH}_{2} \mathrm{Cl}_{2}$ & MW & 15 & 72 \\
\hline 11 & 30 & $\mathrm{EtOH} / \mathrm{H}_{2} \mathrm{O}(1 / 1)$ & MW & 15 & 92 \\
\hline 12 & 30 & $\mathrm{H}_{2} \mathrm{O}$ & Reflux & 45 & 80 \\
\hline 13 & 30 & $\mathrm{EtOH} / \mathrm{H}_{2} \mathrm{O}(1 / 1)$ & Reflux & 25 & 67 \\
\hline 14 & 30 & $\mathrm{EtOH} / \mathrm{H}_{2} \mathrm{O}(1 / 1)$ & Reflux & 35 & 74 \\
\hline 15 & 30 & $\mathrm{EtOH} / \mathrm{H}_{2} \mathrm{O}(1 / 1)$ & Reflux & 45 & 87 \\
\hline 16 & 30 & $\mathrm{EtOH} / \mathrm{H}_{2} \mathrm{O}(1 / 1)$ & Reflux & 60 & 92 \\
\hline 17 & 30 & Solvent free & MW & 60 & 38 \\
\hline
\end{tabular}

After obtaining optimal reaction conditions, in order to produce the corresponding products in high yields and in short reaction times, various aromatic aldehydes were reacted with malononitrile and barbituric acid in synthesis of pyranopyrimidinone derivatives and also, with $\beta$-naphtol in synthesis of xanthene derivatives. The obtained results are summarized in tables 3 and 4.
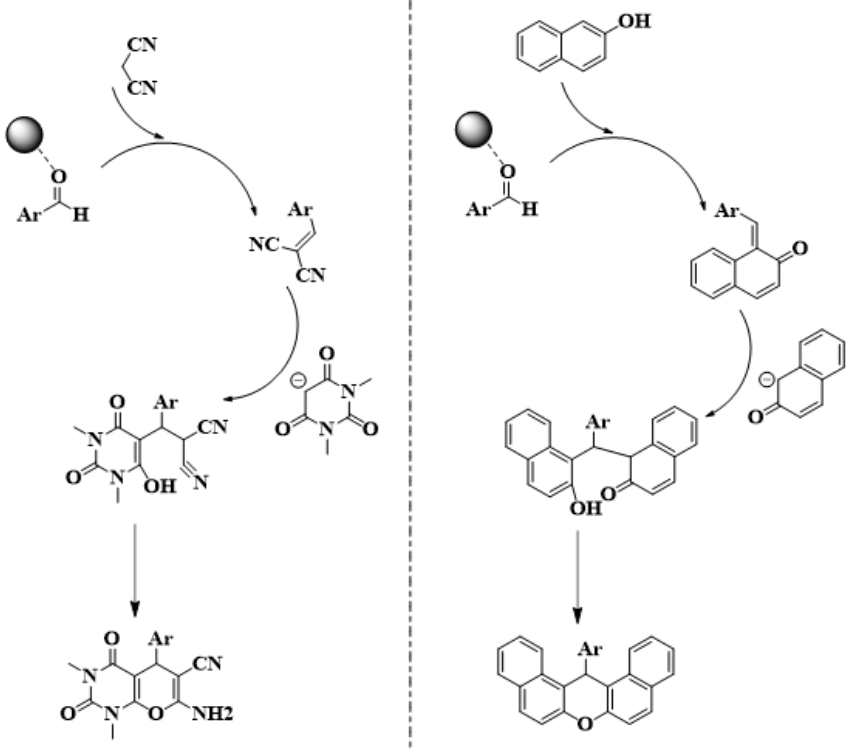

Scheme 3. proposed mechanism for the synthesis of pyranopyrimidinoneand xanthene derivatives in the presence of $\mathrm{ZnO}$-starch nanocomposite. 
J. Chil. Chem. Soc., 64, N4 (2019)

Table 3. Preparation of pyranopyrimidinone derivatives using $\mathrm{ZnO}$-starch nanocomposite.

\begin{tabular}{|c|c|c|c|c|c|c|}
\hline \multirow{2}{*}{ Entry } & \multirow{2}{*}{ Structure } & \multirow{2}{*}{ Time (min) } & \multirow{2}{*}{ Yield (\%) } & \multicolumn{2}{|r|}{$\mathbf{m p}$} & \multirow{2}{*}{ Product } \\
\hline & & & & Found & Reported (Ref) & \\
\hline 1 & & 12 & 91 & $230-231$ & $235-237[38-40]$ & $4 a$ \\
\hline 2 & & 13 & 92 & $240-241$ & $245-247$ [41] & $4 \mathrm{~b}$ \\
\hline 3 & & 14 & 85 & $240-241$ & $247-249$ [42] & $4 c$ \\
\hline 4 & & 19 & 81 & $240-242$ & $250-251$ [43] & $4 d$ \\
\hline 5 & & 11 & 95 & $254-256$ & $265-266$ [43] & $4 \mathrm{e}$ \\
\hline 6 & & 18 & 85 & $241-242$ & 238-239 [44] & $4 \mathrm{f}$ \\
\hline 7 & & 17 & 90 & $158-160$ & $165-166$ [45] & $4 \mathrm{~g}$ \\
\hline 8 & & 11 & 91 & $239-240$ & $241-242$ [45] & $4 \mathrm{~h}$ \\
\hline 9 & & 12 & 93 & $250-251$ & $260-261$ [46] & $4 \mathrm{i}$ \\
\hline
\end{tabular}

Table 4. Preparation of xanthene derivatives using $\mathrm{ZnO}$-starch nanocomposite

\begin{tabular}{|c|c|c|c|c|c|c|}
\hline \multirow{2}{*}{ Entry } & \multirow{2}{*}{ Structure } & \multirow{2}{*}{ Time (min) } & \multirow{2}{*}{ Yield (\%) } & \multicolumn{2}{|r|}{$\mathbf{m p}$} & \multirow{2}{*}{ Product } \\
\hline & & & & Found & Reported (Ref) & \\
\hline 1 & & 14 & 88 & $251-252$ & 255-257 [47] & $7 \mathrm{a}$ \\
\hline 2 & & 15 & 91 & $181-183$ & 179-181 [48] & $7 b$ \\
\hline 3 & & 12 & 92 & $184-185$ & $187-189$ [49] & $7 \mathrm{c}$ \\
\hline 4 & & 17 & 88 & $303-305$ & $310-312$ [50] & $7 d$ \\
\hline 5 & & 17 & 95 & $213-215$ & $217-220[50]$ & $7 \mathrm{e}$ \\
\hline 6 & & 11 & 95 & $197-200$ & 190-192 [51] & $7 f$ \\
\hline 7 & & 14 & 90 & $288-290$ & 295-297 [52] & $7 \mathrm{~g}$ \\
\hline 8 & & 13 & 89 & $215-217$ & 219-221 [53] & $7 \mathrm{~h}$ \\
\hline 9 & & 14 & 88 & $217-220$ & $225-227$ [53] & $7 \mathrm{i}$ \\
\hline 10 & & 12 & 85 & $198-200$ & 201-202 [54] & $7 \mathrm{j}$ \\
\hline 11 & & 15 & 89 & $255-258$ & $265-266$ [55] & $7 \mathrm{k}$ \\
\hline
\end{tabular}




\section{Recycling and Reusing of the nanocatalyst}

One of the most important features of a heterogeneous catalyst is its ability to recycle and reuse it. In order to investigate the recyclability of $\mathrm{ZnO}-\mathrm{Ch} \mathrm{NCs}$, after completion of the reaction, the catalyst was filtered and washed well with plenty of water and acetone. The results showed that the catalyst activity has maintained its activity after four runs (Figure 3).

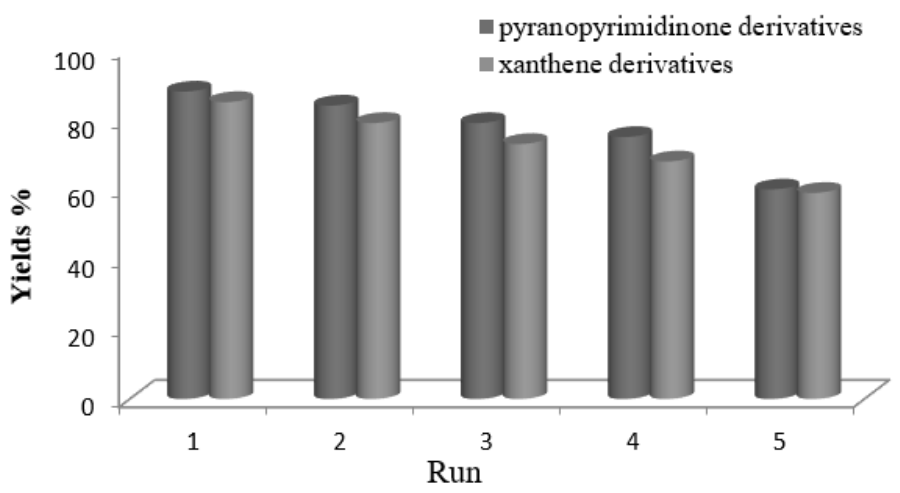

Figure 3. Reusability of nano catalyst in synthesis of pyranopyrimidinone and xanthene derivatives.

\section{Biology}

Antibacterial activity of all of the all synthesized products were evaluated in vitro against Staphylococcus aureus (S. aureus) and Escherichia coli (E. coli), by MIC and MBC method. Tetracycline used as a positive control. All the synthesized products showed good antibacterial activity. According to the results, pyranopyrimidinone derivatives had stronger antibacterial activity against $S$. aureus and E. coli than the xanthene derivatives. The MIC and MBC Results are tabulated in Tables 5 and 6.

Table 5. Results of in vitro antibacterial activity with MIC method against $S$. aureus of synthesized compounds.

\begin{tabular}{|c|c|c|c|c|c|c|c|}
\hline \multirow{2}{*}{ Sample } & \multicolumn{5}{|c|}{ MIC $\boldsymbol{\mu g} / \mathbf{m L}$} & MBC $\boldsymbol{\mu g} / \mathbf{m L}$ \\
\cline { 2 - 8 } & $\mathbf{2 0 0 0}$ & $\mathbf{1 0 0 0}$ & $\mathbf{5 0 0}$ & $\mathbf{2 5 0}$ & $\mathbf{1 2 5}$ & $\mathbf{6 2 . 5}$ & \\
\hline 4a & Inhibit. & Inhibit. & Inhibit. & Inhibit. & Grow. & Grow. & 250 \\
\hline 4b & Inhibit. & Inhibit. & Inhibit. & Inhibit. & Grow. & Grow. & 250 \\
\hline 4c & Inhibit. & Inhibit. & Inhibit. & Inhibit. & Grow. & Grow. & 250 \\
\hline 4d & Inhibit. & Inhibit. & Inhibit. & Inhibit. & Grow. & Grow. & 250 \\
\hline 4e & Inhibit. & Inhibit. & Inhibit. & Inhibit. & Grow. & Grow. & 250 \\
\hline 4f & Inhibit. & Inhibit. & Inhibit. & Inhibit. & Grow. & Grow. & 250 \\
\hline 4g & Inhibit. & Inhibit. & Inhibit. & Inhibit. & Grow. & Grow. & 250 \\
\hline 4h & Inhibit. & Inhibit. & Inhibit. & Inhibit. & Grow. & Grow. & 250 \\
\hline 4i & Inhibit. & Inhibit. & Inhibit. & Inhibit. & Grow. & Grow. & 250 \\
\hline 7a & Inhibit. & Inhibit. & Inhibit. & Grow. & Grow. & Grow. & 500 \\
\hline 7b & Inhibit. & Inhibit. & Inhibit. & Grow. & Grow. & Grow. & 500 \\
\hline 7c & Inhibit. & Inhibit. & Inhibit. & Grow. & Grow. & Grow. & 500 \\
\hline 7d & Inhibit. & Inhibit. & Inhibit. & Grow. & Grow. & Grow. & 500 \\
\hline 7e & Inhibit. & Inhibit. & Inhibit. & Grow. & Grow. & Grow. & 500 \\
\hline 7f & Inhibit. & Inhibit. & Inhibit. & Grow. & Grow. & Grow. & 500 \\
\hline 7g & Inhibit. & Inhibit. & Inhibit. & Grow. & Grow. & Grow. & 500 \\
\hline 7h & Inhibit. & Inhibit. & Inhibit. & Grow. & Grow. & Grow. & 500 \\
\hline 7i & Inhibit. & Inhibit. & Inhibit. & Grow. & Grow. & Grow. & 500 \\
\hline 7j & Inhibit. & Inhibit. & Inhibit. & Grow. & Grow. & Grow. & 500 \\
\hline 7k & Inhibit. & Inhibit. & Inhibit. & Grow. & Grow. & Grow. & 500 \\
\hline Tetracycline & Inhibit. & Inhibit. & Inhibit. & Inhibit. & Inhibit. & Inhibit. & $<62.5$ \\
\hline
\end{tabular}

Table 6. Results of in vitro antibacterial activity with MIC method against $E$. coli of synthesized compounds.

\begin{tabular}{|c|c|c|c|c|c|c|c|}
\hline \multirow{2}{*}{ Sample } & \multicolumn{6}{|c|}{$\mathrm{MIC} \mu \mathrm{g} / \mathrm{mL}$} & \multirow{2}{*}{$\mathrm{MBC} \mu \mathrm{g} / \mathrm{mL}$} \\
\hline & 2000 & 1000 & 500 & 250 & 125 & 62.5 & \\
\hline $4 \mathrm{a}$ & Inhibit. & Inhibit. & Inhibit. & Grow. & Grow. & Grow. & 500 \\
\hline $4 \mathrm{~b}$ & Inhibit. & Inhibit. & Inhibit. & Grow. & Grow. & Grow. & 500 \\
\hline $4 \mathrm{c}$ & Inhibit. & Inhibit. & Inhibit. & Grow. & Grow. & Grow. & 500 \\
\hline $4 \mathrm{~d}$ & Inhibit. & Inhibit. & Grow. & Grow. & Grow. & Grow. & 1000 \\
\hline $4 \mathrm{e}$ & Inhibit. & Inhibit. & Grow. & Grow. & Grow. & Grow. & 1000 \\
\hline $4 \mathrm{f}$ & Inhibit. & Inhibit. & Grow. & Grow. & Grow. & Grow. & 1000 \\
\hline $4 \mathrm{~g}$ & Inhibit. & Inhibit. & Grow. & Grow. & Grow. & Grow. & 1000 \\
\hline $4 \mathrm{~h}$ & Inhibit. & Inhibit. & Grow. & Grow. & Grow. & Grow. & 1000 \\
\hline $4 \mathrm{i}$ & Inhibit. & Inhibit. & Grow. & Grow. & Grow. & Grow. & 1000 \\
\hline $7 \mathrm{a}$ & Inhibit. & Inhibit. & Grow. & Grow. & Grow. & Grow. & 1000 \\
\hline $7 \mathrm{~b}$ & Inhibit. & Inhibit. & Grow. & Grow. & Grow. & Grow. & 1000 \\
\hline $7 \mathrm{c}$ & Inhibit. & Inhibit. & Grow. & Grow. & Grow. & Grow. & 1000 \\
\hline $7 \mathrm{~d}$ & Inhibit. & Inhibit. & Grow. & Grow. & Grow. & Grow. & 1000 \\
\hline $7 \mathrm{e}$ & Inhibit. & Inhibit. & Grow. & Grow. & Grow. & Grow. & 1000 \\
\hline $7 \mathrm{f}$ & Inhibit. & Inhibit. & Grow. & Grow. & Grow. & Grow. & 1000 \\
\hline $7 \mathrm{~g}$ & Inhibit. & Inhibit. & Grow. & Grow. & Grow. & Grow. & 1000 \\
\hline $7 \mathrm{~h}$ & Inhibit. & Inhibit. & Grow. & Grow. & Grow. & Grow. & 1000 \\
\hline $7 \mathrm{i}$ & Inhibit. & Inhibit. & Inhibit. & Grow. & Grow. & Grow. & 500 \\
\hline $7 \mathrm{j}$ & Inhibit. & Inhibit. & Inhibit. & Grow. & Grow. & Grow. & 500 \\
\hline $7 \mathrm{k}$ & Inhibit. & Inhibit. & Inhibit. & Grow. & Grow. & Grow. & 500 \\
\hline Tetracycline & Inhibit. & Inhibit. & Inhibit. & Inhibit. & Inhibit. & Inhibit. & $<62.5$ \\
\hline
\end{tabular}

Figure 6 shows an overview of the steps to perform the MIC test on product 4a against $\mathrm{S}$. aureus (positive germ) and E. coli (negative geram).
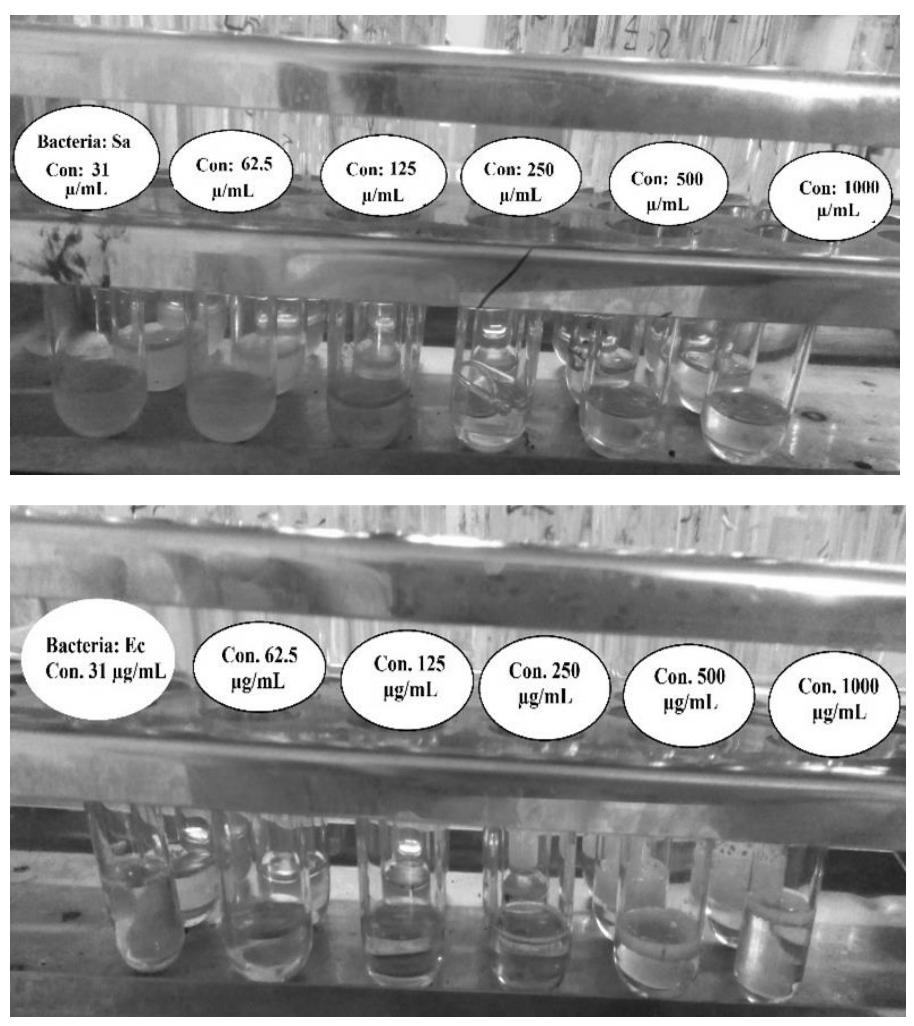

Figure 6. Perform MIC test on product 4a and against S. aureus (gram positive) and E. coli (gram negative). 


\section{EXPERIMENTAL}

\section{Material and Instrumentation}

The chemicals used in the synthesis of the pyranopyrimidinone and xanthene derivatives were obtained from the Merck and Aldrich Chemical Co. All chemicals and solvents were analytical reagent grade. Reactions were monitored by thin layer chromatography on $0.2 \mathrm{~mm}$ silica gel F-252 (Merck) plates. Melting points were determined by open capillary Electrothermal $9100 \mathrm{~s}$ and were uncorrected. ${ }^{1} \mathrm{H}(400 \mathrm{MHz})$ and ${ }^{13} \mathrm{C}(100 \mathrm{MHz})$ spectra were recorded on Bruker 3000 NMR spectrometer in $\mathrm{CDCl}_{3} / \mathrm{DMSO}-d^{6}$ (with TMS as internal reference). Ultrasound device Astra 3D ( $9.5 \mathrm{~L}, 45 \mathrm{kHz}$ frequency, input power with heating, $305 \mathrm{~W}$, number of transducers, 2) from TECNO-GAZ was used. All the reactions were carried out in a microwave oven.

\section{Preparation of ZnO-Chitosan nanocomposite (ZnO\&Ch NCs)}

The synthesis of the ZnO\&Ch NCs according to the articles was done with some changes [56]. first $0.75 \mathrm{~g}$ of $\mathrm{ZnO}$ powder $(9.2 \mathrm{mmol})$ was dissolved in 100 $\mathrm{mL}$ of $1 \%$ acetic acid solution. The solution is sonicated for $10 \mathrm{~min}$. After this time $1 \mathrm{~g}$ of chitosan was added to the solution. The mixture was sonicated for 30 min. After this time, added a drop by drop of $1 \mathrm{M} \mathrm{NaOH}$ solution to the mixture until the solution attained $\mathrm{pH} 10$. The solution was heated in microwave oven for about $10 \mathrm{~min}$. The mixture was then filtered and washed with distilled water several times then dried in an oven at $50^{\circ} \mathrm{C}$ for $1 \mathrm{hr}$.

\section{General procedure for the preparation of pyrano-pyrimidinones}

A mixture of the aromatic aldehyde $(1 \mathrm{mmol})$, malononitrile $1.2 \mathrm{mmol}(\sim 80$ $\mathrm{mg}$ ), barbituric acid $1 \mathrm{mmol}(156 \mathrm{mg})$ in the presence of $28 \mathrm{mg} \mathrm{ZnO}-\mathrm{Ch} \mathrm{NCs}$ in $\mathrm{H}_{2} \mathrm{O}: \mathrm{EtOH}(2 \mathrm{ml}: 2 \mathrm{ml})$ was irradiated in a microwave oven at a power of $230 \mathrm{~W}$. After completion of the reaction, the resulting solid (crude product) was filtered and then recrystallized from EtOH to obtain pure product. The physical data (m.p., NMR, IR) of these known compounds were found to identical with those reported in the literature.

\section{Spectral data of selected compounds are given below;}

7-amino-5-(3-chlorophenyl)-2,4-dioxo-1,3,4,5-tetrahydro-2H-pyrano[2,3d]pyrimidine-6-carbonitrile (4b); Dark yellow powder, m.p. 240-241 ${ }^{\circ} \mathrm{C}$. IR $(\mathrm{KBr})\left(v_{\max } / \mathrm{cm}^{-1}\right): 3419,3300\left(\mathrm{NH}_{2}\right), 3186(\mathrm{NH}), 2192(\mathrm{C} \equiv \mathrm{N}), 1689$ and 1654 $(\mathrm{C}=\mathrm{O}) .{ }^{1} \mathrm{HNMR}: \delta=4.47(\mathrm{~s}, 1 \mathrm{H}, \mathrm{H}-5), 7.28\left(\mathrm{broad}, 2 \mathrm{H}, \mathrm{H}-\mathrm{Ar}, \mathrm{NH}_{2}\right), 7.61(\mathrm{t}, 1 \mathrm{H}$, $\left.{ }^{3} \mathrm{~J}=7.6 \mathrm{~Hz}, \mathrm{H}-\mathrm{Ar}\right), 7.74\left(\mathrm{~d}, 1 \mathrm{H},{ }^{3} \mathrm{~J}=7.6 \mathrm{~Hz}, \mathrm{H}-\mathrm{Ar}\right), 7.61\left(\mathrm{t}, 1 \mathrm{H},{ }^{3} \mathrm{~J}=7.6 \mathrm{~Hz}, \mathrm{H}-\mathrm{Ar}\right)$, 8.06 (broad, 1H, H-Ar), 8.08-8.13 (m, 1H, H-Ar), 11.11 (s, 1H, NH), 12.16 (brs, $1 \mathrm{H}, \mathrm{NH}) \mathrm{ppm}$.

7-amino-5-(2-chlorophenyl)-2,4-dioxo-1,3,4,5-tetrahydro-2H-pyrano[2,3d]pyrimidine-6-carbonitrile (4c); Dark yellow powder, m.p. 240-241 ${ }^{\circ} \mathrm{C}$. IR $(\mathrm{KBr})\left(v_{\max } / \mathrm{cm}^{-1}\right): 3410,3370\left(\mathrm{NH}_{2}\right), 3158(\mathrm{NH}), 2185(\mathrm{C} \equiv \mathrm{N}), 1689(\mathrm{C}=\mathrm{O})$; ${ }^{1} \mathrm{HNMR}$ : $\delta=4.72$ (s, $\left.1 \mathrm{H}, \mathrm{H}-5\right), 7.153$ (broad, $\left.2 \mathrm{H}, \mathrm{NH}_{2}\right), 7.208-7.281(\mathrm{~m}, 3 \mathrm{H}, \mathrm{H}-$ Ar), 7.36 (d, 1H, H-Ar), 11.06 (s, 1H, NH), 12.08 (board, 1H, NH) ppm.

7-amino-5-(2,3-dichlorophenyl)-2,4-dioxo-1,3,4,5-tetrahydro-2Hpyrano[2,3-d]pyrimidine-6-carbonitrile (4d); White color powder, m.p. 240$242{ }^{\circ} \mathrm{C}$. IR $(\mathrm{KBr})\left(v_{\max } / \mathrm{cm}^{-1}\right): 3401,3385\left(\mathrm{NH}_{2}\right), 3255(\mathrm{NH}), 2225(\mathrm{C} \equiv \mathrm{N}), 1688$, $1642(\mathrm{C}=\mathrm{O})$

7-amino-5-(4-nitrophenyl)-2,4-dioxo-1,3,4,5-tetrahydro-2H-pyrano[2,3d]pyrimidine-6-carbonitrile (4h); White color powder, m.p. $239-240^{\circ} \mathrm{C}$. IR $(\mathrm{KBr})\left(v_{\max } / \mathrm{cm}^{-1}\right): 3477,3238\left(\mathrm{NH}_{2}\right), 3127(\mathrm{NH}), 2192(\mathrm{C} \equiv \mathrm{N}), 1677,1645$ $(\mathrm{C}=\mathrm{O}) ;{ }^{1} \mathrm{H}-\mathrm{NMR}:{ }^{\delta}=4.42(\mathrm{~s}, 1 \mathrm{H}), 7.21$ (broad, $\left.2 \mathrm{H}, \mathrm{NH}_{2}\right), 7.52\left(\mathrm{~d}, 2 \mathrm{H},{ }^{3} \mathrm{JHH}=8.8\right.$ $\mathrm{Hz}, \mathrm{H}-\mathrm{Ar}$ ), 8.16 (d, 2H, $\left.{ }^{3} \mathrm{JHH}=8.8 \mathrm{~Hz}, \mathrm{H}-\mathrm{Ar}\right), 11.12$ (brs, $\left.1 \mathrm{H}, \mathrm{NH}\right), 12.17$.

\section{General procedure for the synthesis of tetrahydrobenzo[a]xanthen}

$288 \mathrm{mg}$ of $\beta$-naphthol ( $2 \mathrm{mmol})$, benzaldehyde $(1 \mathrm{mmol})$ and $30 \mathrm{mg} \mathrm{ZnO}-\mathrm{Ch}$ $\mathrm{NCs}$ were added to the reaction vessel containing $\mathrm{H}_{2} \mathrm{O}: \mathrm{EtOH}(2 \mathrm{ml}: 2 \mathrm{ml})$. The solution was irradiated in a microwave oven at a power of $230 \mathrm{~W}$. The progress of reactions monitored by TLC. After completion of the reaction, the reaction mixture was cooled to room temperature. Then the catalyst was separated by filtration. Finally, the crude product was recrystallized from $\mathrm{EtOH}$ to yield pure xanthene derivatives. All products were characterized by comparison of their melting point and FT-IR spectra with references. Some of them were ${ }^{1}$ HNMR spectral to prove the structure of the compounds.

\section{Spectral data of selected compounds are given below;}

14-phenyl-14H-dibenzo[a,j]xanthene (7c); White solid, mp 184-185 ${ }^{\circ} \mathrm{C}$, IR $\left(\mathrm{KBr}, \mathrm{cm}^{-1}\right): v_{\max }=3069,3041,2927,1614,1581,1508,1474,1361 ;{ }^{1} \mathrm{H}$ NMR
(400 MHz, CDCl3): $\delta=6.53$ (s, 1H, CH), 7.045 (t, J = 7.2 Hz, 1H, ArH), 7.183 $(\mathrm{t}, \mathrm{J}=7.2 \mathrm{~Hz}, 2 \mathrm{H}, \mathrm{ArH}), 7.450(\mathrm{t}, \mathrm{J}=7.5 \mathrm{~Hz}, 2 \mathrm{H}, \mathrm{ArH}), 7.53-7.60(\mathrm{~m}, 6 \mathrm{H}, \mathrm{ArH})$, $7.621(\mathrm{t}, \mathrm{J}=8 \mathrm{~Hz}, 4 \mathrm{H}, \mathrm{ArH}), 8.437(\mathrm{~d}, \mathrm{~J}=8 \mathrm{~Hz}, 2 \mathrm{H}, \mathrm{ArH}) \mathrm{ppm}$

14-(3-methylphenyl)-14H-dibenzo[a,j]xanthene (7f); Yellow solid,mp 197$200{ }^{\circ} \mathrm{C}, \mathrm{IR}\left(\mathrm{KBr}, \mathrm{cm}^{-1}\right): v_{\max }=3018,2921,1623,1591,1512,1457,1400,1249$, 960, 806, 806, 743; ${ }^{1} \mathrm{H}$ NMR (400 MHz,CDCl3): $\delta=2.074$ (s, 3H, CH3), 6.67 (s, $1 \mathrm{H}, \mathrm{CH}), 6.77(\mathrm{~d}, \mathrm{~J}=7.6 \mathrm{~Hz}, 1 \mathrm{H}, \mathrm{ArH}), 7.04(\mathrm{t}, \mathrm{J}=7.6 \mathrm{~Hz}, 1 \mathrm{H}, \mathrm{ArH}), 7.312$ $(\mathrm{s}, 1 \mathrm{H}, \mathrm{CH}), 7.454(\mathrm{~m}, 2 \mathrm{H}, \mathrm{ArH}), 7.537(\mathrm{~s}, 1 \mathrm{H}, \mathrm{CH}), 7.56(\mathrm{~d}, \mathrm{~J}=8.8 \mathrm{~Hz}, 2 \mathrm{H}, \mathrm{ArH})$ $7.630(\mathrm{~m}, 2 \mathrm{H}, \mathrm{ArH}) \mathrm{ppm}, 7.908-7.936(\mathrm{~m}, 4 \mathrm{H}, \mathrm{ArH}) \mathrm{ppm}, 8.671(\mathrm{~d}, \mathrm{~J}=8.4 \mathrm{~Hz}$, $2 \mathrm{H}, \mathrm{ArH}) \mathrm{ppm}$.

14-(4-methoxyphenyl)-14H-dibenzo[a,j]xanthene (7h); White solid, $\mathrm{mp} 215$ $217^{\circ} \mathrm{C}$. IR $\left(\mathrm{KBr}, \mathrm{cm}^{-1}\right): v_{\max }=3072,2834,1622,1592,1509,1458,1438,1399$, 1249, 1082. ${ }^{1} \mathrm{H}$ NMR $(400 \mathrm{MHz}, \mathrm{CDCl} 3): \delta=3.377$ (s, 3H, CH3), $6.68(\mathrm{~s}, 1 \mathrm{H}$, $\mathrm{CH}), 6.69(\mathrm{~d}, \mathrm{~J}=8.4 \mathrm{~Hz}, 2 \mathrm{H}, \mathrm{ArH}), 7.456(\mathrm{t}, \mathrm{J}=7.2 \mathrm{~Hz}, 2 \mathrm{H}, \mathrm{ArH}), 7.53(\mathrm{t}, \mathrm{J}=$ $7.5 \mathrm{~Hz}, 4 \mathrm{H}, \mathrm{ArH}), 7.621(\mathrm{t}, 2 \mathrm{H}, \mathrm{ArH}), 7.926(\mathrm{t}, \mathrm{J}=8.4 \mathrm{~Hz}, 4 \mathrm{H}, \mathrm{ArH}), 8.66(\mathrm{~d}, \mathrm{~J}$ $=8.4 \mathrm{~Hz}, 2 \mathrm{H}, \mathrm{ArH}) \mathrm{ppm}$

14-(3- chlorophenyl)-14H-dibenzo[a,j]xanthene (7i); White solid, mp 217 $220^{\circ} \mathrm{C}$. IR $\left(\mathrm{KBr}, \mathrm{cm}^{-1}\right): v_{\max }=3467,3057,1658,1622,1591,1514,1473,1458$, 1431, 1400, 1240, 1238. ${ }^{1} \mathrm{H}$ NMR (400 MHz, CDCl3): $\delta=6.73$ (s, 1H, CH), 7.00 (t, J = 8.4 Hz, 1H, ArH), $7.07(\mathrm{t}, \mathrm{J}=7.2 \mathrm{~Hz}, 1 \mathrm{H}, \mathrm{ArH}), 7.31(\mathrm{~d}, \mathrm{~J}=8 \mathrm{~Hz}, 1 \mathrm{H}, \mathrm{ArH})$ 7.43 (s, 1H, ArH), 7.46 (t, 2H, ArH), $7.53(\mathrm{~d}, \mathrm{~J}=8.4 \mathrm{~Hz}, 2 \mathrm{H}, \operatorname{ArH}), 7.62$ (d, J = $7.2 \mathrm{~Hz}, 2 \mathrm{H}, \mathrm{ArH}), 7.93$ (d, J= $8.4 \mathrm{~Hz}, 4 \mathrm{H}, \mathrm{ArH}), 8.59$ (d, J=8.4, 2H, ArH) ppm.

14-(3-bromophenyl)-14H-dibenzo[a,j]xanthene (7j); White solid, $\mathrm{mp} 198$ $200^{\circ} \mathrm{C}$. IR $\left(\mathrm{KBr}, \mathrm{cm}^{-1}\right): v_{\max }=3065,2923,1622,1590,1566,1514,1472,1456$, $1432,1392,1239 .{ }^{1} \mathrm{H}$ NMR $(400 \mathrm{MHz}, \mathrm{CDCl} 3): \delta=6.76(\mathrm{~s}, 1 \mathrm{H}, \mathrm{CH}), 7.11(\mathrm{t}, \mathrm{J}$ $=7.6 \mathrm{~Hz}, 1 \mathrm{H}, \mathrm{ArH}), 7.17-7.19(\mathrm{~m}, 1 \mathrm{H}, \mathrm{ArH}), 7.47(\mathrm{t}, \mathrm{J}=7.2 \mathrm{~Hz}, 2 \mathrm{H}, \mathrm{ArH}), 7.57$ (d, $2 \mathrm{H}, \mathrm{J}=8.8 \mathrm{~Hz}, \mathrm{ArH}), 7.63-7.67$ (m, 3H, ArH), 7.81 (broad, 1H, ArH), 7.94 $(\mathrm{d}, \mathrm{J}=8.8 \mathrm{~Hz}, 4 \mathrm{H}, \mathrm{ArH}), 8.70(\mathrm{~d}, \mathrm{~J}=8.4,2 \mathrm{H}, \mathrm{ArH}) \mathrm{ppm}$.

\section{Recycling and reusing of the nanocatalyst}

The catalyst used after the completion of the reaction is filtered and washed with plenty of water and acetone and then dried at $70{ }^{\circ} \mathrm{C}$ for $8 \mathrm{~h}$. The reusability of the catalyst was tested by repeating the reaction in its optimal conditions. The results showed that the catalytic activity of the $\mathrm{ZnO}-\mathrm{Ch} \mathrm{NCs}$ did not decrease significantly even after four catalytic cycles.

\section{Biology}

\section{MIC assay}

To prepare a liquid culture medium, first $0.8 \mathrm{gr}$ of Nutrient Broth was added into $100 \mathrm{ml}$ of distilled water and then $1 \mathrm{ml}$ of it was added into the test tube. The test tubes were autoclaved at $115{ }^{\circ} \mathrm{C}$ for 15 minutes.

Six concentrations of each sample were prepared $(1000,500,250,125,62.5$, $31.25 \mu \mathrm{g} / \mathrm{ml}$ ) in DMSO. $1 \mathrm{ml}$ of samples were added to the autoclaved test tubes. The concentration of the standard bacteria $(0.5 \mathrm{McF}$ arland standard) was made. $100 \mu \mathrm{l}$ of each bacterium was poured into each the test tube. The tubes were incubating overnight. Broth tubes that appear turbid are indicative of bacterial growth while tubes that remain clear indicate no growth. The MIC of the antibiotic is the lowest concentration that does not show growth.

\section{MBC assay}

The concentration of sample which showed MIC and two higher concentrations were applied to MBC test. The concentration in which the bacterium has not grown is reported as MBC.

\section{CONCLUSION}

In conclusion this paper describes a convenient and efficient process for the synthesis of corresponding pyranopyrimidinone and xanthene derivatives in the presence of $\mathrm{ZnO}-\mathrm{Ch} \mathrm{NCs}$ under microwave radiation. The protocol used in this research has many advantages such as reduced time, low cost, the simplicity of using the catalyst, separating the catalyst only by filtering, recyclating the catalyst, simple processing. In many cases, the products crystallized directly from the reaction mixture in high purity. In general, in this method, aldehydes bearing electron-donating groups require more time to complete the reaction, but the rate of formation of the product in aldehydes with electron-withdrawing groups will be higher. It was observed that $\mathrm{ZnO}-\mathrm{Ch} \mathrm{NCs}$ catalyzed both types of reactions well under microwave irradiation and green solvent to the formation of the corresponding pyranopyrimidinone and xanthene derivatives. 


\section{REFERENCES}

1. A. K. Bhattachary, K. C. Rana, Mendeleev Commun. 17, 247, (2007).

2. M. T. Maghsoodlou, R. Heydari, M. Lashkari, F. Mohamadpour, Indian J Chem. 56B, 160, (2017).

3. M. Seyyedhamzeh, M. Mirzaei, A. Bazgir, Dyes Pigm. 76, 836, (2008).

4. R. Gupta, A. Jain, R. Joshi, M. Jain, Bull Korean Chem Soc. 32, 899, (2011).

5. M. M. Hanna, Eur J Med Chem. 55, 12, (2012).

6. S. V. Shinde, W. N. Jadhav, N. N. Karade, Orient J Chem. 26, 307, (2010).

7. S. Gupta, P. Gupta, A. Sachar, R. L. Sharma, Indian J Chem, 49, 1243, (2010).

8. Z. Karimi-Jaberi, M. M. Hashemi, Monatsh Chem. 139, 605, (2008).

9. W. Su, D. Yang, C. Jin, B. Zhang, Tetrahedron Lett. 49, 3391, (2008).

10. J. Q. Wang, R. G. Harvey, Tetrahedron. 58, 5927, (2002).

11. A. Jha, J. Beal, Tetrahedron Lett. 45, 8999, (2004).

12. R. J. Sarma, J. B. Baruah, Dyes Pigm. 64, 91, (2005).

13. S. Kobayashi, T. Busujima, S. Nagayama, Chem Eur J. 6, 3491, ( 2000)

14. M. G. Constantino, V. Lacerda, Synth. Commun. 37, 3529, (2007).

15. C. K. Z. Andrade, Curr Org Synth. 1, 333, (2004).

16. P. Kulkarni, J Mex Chem Soc. 62, 1, (2018).

17. K. Gong, D. Fang, H. L. Wang, X. L. Zhou, Z. L. Liu, Dyes Pigm. 80, 30, (2009).

18. D. Liu, S. Zhou, J. Gao, L. Li, D. Xu, J Mex Chem Soc. 57, 345, (2013).

19. A. Rahmatpour, Monatsh Chem. 142, 1259, (2011).

20. R. Kumar, G. C. Nandi, R. K. Verma, M. S. Singh, Tetrahedron Lett. 51, 442, (2010)

21. J. Q. Wang, R. G. Harvey, Tetrahedron. 58, 5927, (2002)

22. H. Stetler, W. Forest, Academic Press NY. 2, 51, (1963).

23. S. Ko, C. F. Yao, Tetrahedron Lett. 47, 8827, (2006).

24. K. Gong, D. Fang, H. L. Wang, X. L. Zhou, Z. L. Liu, Dyes Pigm. 80, 30, (2009).

25. M. H. Majid, A. Hamideh, B. Khadijeh, S. Mina, A. O. Hossein, F. B. Fatemeh, Bull Chem Soc Ethiop. 25, 399, (2011).

26. B. L. Hayes, Microwave Synthesis Chemistry at the Speed of Light, CEM Publishing, 2002.

27. N. E. Leadbeater, Microwave Heating as a Tool for Sustainable Chemistry, CRC Press, 2010.

28. J. P. Tierney, P. Lidstroem, Microwave Assisted Organic Synthesis. CRC Press, 2005.

29. C. O. Kappe, A. Stadler, D. Dallinger, Microwave in Organic and Medicinal Chemistry. Wiley-VCH-Verlag, 2012.

30. F. Smith, W. Kenneth, A. Humera, B. Lorraine, L. Laberge, J. Rousell, Tetrahedron Lett. 27, 279, (1986).

31. L. Jin, Y. Zhang, L. Yan, Y. Guo, L. Niu, Molecules. 17, 9361, (2012).

32. S. A. H. Vahabi, F. Hatamjafari, K. Pourshamsian, $O J$ C. 30, 849, (2014).

33. N. Montazeri, K. Pourshamsian, H. rezaei, M. Fouladi, Asian J Chem. 25, $3463,(2013)$

34. N. Montazeri, K. Pourshamsian, M. Bayazi, S. Kabiri, Asian J Chem. 25, $3373,(2013)$

35. N. Montazeri, K. Pourshamsian, M. Rahgol, M. Bayazi, Asian J Chem. 24, $5361,(2012)$

36. N. Montazeri, K. Pourshamsian, A. Fomani, S. J. Kalantarian, Asian J Chem. 24, 2805, (2012)

37. K. Pourshamsian, N. Montazeri, K. Rad-Moghadam, S. A. Asgari, J Heterocycl Chem. 47, 1439, (2010).

38. M. A. Pasha, M. Krishnappa, V. P. Jayashankara, Indian J Chem. 49B, 1428, (2010).

39. V. K. Alhuwalia, R. A, Batla, Khurana, R. Kumar, Indian J Chem Sec-B. 29 , $1141,(1990)$.

40. M. Jinxia, Z. Wenhua, T. Yajun, W. Zhiguo, Nanoscale Res Lett. 11, 1, (2016).

41. J. Albadi, A. Mansournezhad, T. Sadeghi, Res Chem Intermed. 41, 8317, (2015).

42. B. Sabour, M. H. Peyrovi, M. Hajimohammadi,, Res Chem Intermed. 41, 1343, (2015).

43. N. Sheikhan-Shamsabadi, M. Ghashang, Main Group Metal Chemistry. 40, 19, (2017).

44. A. R. Bhat, A. H. Shalla, R. S. Dongre, J. Taibah, J Univ Sci. 10, 9, (2016).

45. A. Mobinikhaledi, M. A. Bodaghi, Acta Chim. Slov. 57, 931, (2010).

46. H. Kefayati, M. Valizadeh, A. Islamnezhad, Anal Bioanal Electrochem. 6, $80,(2014)$.

47. G. H. Mahdavinia, S. Rostamizadeh, A. M. Amani, Z. Emdadi, Ultrason Sonochem. 16, 7, (2009).

48. K. Gong, D. Fang, H. L. Wang, X. L. Zhou, Z. L. Liu, Dyes Pigm. 80, 30, (2009).
49. B. B. F. Mirjalili, A. Bamoniri, A. Akbari, Tetrahedron Lett. 49, 6454, (2008).

50. P. Kumari, V. Yathindranath, M. S. Chauhan, Synth. Commun. 38, 637, (2008).

51. M. A. Pasha, V. P. Jayashankara, Bioorg Med. Chem. Lett. 17, 621, (2007).

52. J .V. Madhav, B. S. Kuarm, B. Rajitha, ARKIVOC. 2, 204, (2008).

53. F.Q. Ding, L.T. An, J. P. Zou, Chinese J Chem. 25, 645, (2007)

54. J. P. Poupelin, G. Saint-Ruf, O. Foussard-Blanpin, G. Narcisse, G. UchidaErnouf, R. Lacroix, Eur. J. Med. Chem. 13, 67, (1978).

55. S. Rostamizadeh, N. Shadjou, A.M. Amani, S. Balalaie, Chinese Chem Lett, 19, 1151, (2008).

56. M.M. AbdElhady, J Carbohyd Chem. 2012, 1, (2012). 